\title{
La familia Poaceae del distrito de Arahuay (Canta, Lima, Perú)
}

\author{
The family Poaceae from Arahuay district (Canta, Lima, Peru)
}

1 Museo de Historia Natural - Laboratorio de Florística. Universidad Nacional Mayor de San Marcos. Av. Arenales 1256, Jesús María.

Email Paúl Gonzáles:

pGonzálesarce@hotmail.com.

2 Instituto de Investigación de Ciencias Biológicas Antonio Raimondi (ICBAR), Facultad de Ciencias Biológicas, UNMSM.

3 Facultad de Ciencias Naturales y Matemática, Universidad Nacional Federico Villarreal, Calle San Marcos, 351, Lima 21.

$\begin{array}{ll}\text { Presentado: } & 20 / 02 / 2011 \\ \text { Aceptado: } & 30 / 07 / 2011\end{array}$ $\begin{array}{ll}\text { Aceptado: } & \text { 30/07/2011 } \\ \text { Publicado online: } & \text { 25/08/2011 }\end{array}$

\section{Paúl Gonzáles ${ }^{1}$, Eduardo Navarro', María. I. La Torre ${ }^{1,3}$ y Asunción Cano ${ }^{1,2}$}

\section{Resumen}

Se reportan para el distrito de Arahuay (Canta, Lima, Perú) (zona baja y media) un total de 49 especies de la familia Poaceae agrupadas en 28 géneros, 14 tribus y 4 subfamilias. El género Poa es el más diverso con cinco especies, seguido por Eragrostis y Nassella con cuatro especies cada una. Las especies Calamagrostis spiciformis Hack. ex Stuck., Chloris halophila Parodi, Lamprothyrsus peruvianus Hitchc., Festuca glyceriantha Pilg., Lolium multiflorum Lam. y Poa supina Schrad. son nuevos reportes para el departamento de Lima. Se presentan claves dicotómicas para la determinación de los géneros y para las especies en los casos pertinentes. Para cada especie se incluye datos sobre su hábitat, distribución y el material estudiado.

Palabras Claves: Flora, Poaceae, taxonomía, vertientes occidentales, Perú.

\section{Abstract}

We report for the low and half elevations of the Arahuay district (Canta, Lima, Perú), a total of 49 species of the family Poaceae, grouped in 28 genera, 14 tribes and four subfamilies. Poa is the most diverse genus with five species, followed by Eragrostis and Nassella with four species each one. The species Calamagrostis spiciformis, Chloris halophila, Lamprothyrsus peruvianus, Festuca glyceriantha, Lolium multiflorum and Poa supina are new reports for the department of Lima. We provide dichotomic keys for genera and species determination. Additional information about habitat, distribution and the studied material is provided for each species.

Keywords: Flora, Poaceae, taxonomy, western slopes, Peru.

\section{Introducción}

La familia Poaceae está ampliamente distribuida en el mundo, sus especies son componentes principales de varias formaciones vegetales y es una de la familia que más beneficios brindan al hombre; por lo tanto es la de mayor importancia económica y ecológica dentro de las angiospermas. Generalmente son herbáceas o muy raramente leñosas (bambúes), poseen cerca de 750 genéros y 10000 especies (Watson \& Dallwitz, 1992) que se encuentran presentes en todas las latitudes, desde el nivel del mar hasta por encima de los $5000 \mathrm{msnm}$. En la flora peruana, están representadas por alrededor de 700 especies agrupadas en 160 géneros (Tovar 1993; Ulloa et al. 2004), destacando la presencia de la mayoría de las subfamilias existentes en el mundo

La familia Poaceae por su gran distribución ha sido recolectada y estudiada por muchos botánicos, en mayor grado por Tovar (1993), llegándose a la conclusión, que es una de las familias más importantes para el territorio peruano. Ocupa el quinto lugar de familias con mayor número de especies (La Torre et al. 2007; Van Der Werff \& Conciglio 2004), siendo elementos florísticos que están presentes en la mayoría de formaciones vegetales que ocurren en nuestro país (Weberbauer 1945; Brack 1986; Brako \& Zarucchi 1993). Para el departamento de Lima se ha registrado 150 especies (Brako \& Zarucchi 1993), lo que la convierte en la segunda familia con mayor diversidad de especies para este departamento. Además 15 especies son endémicas para este departamento (La Torre et al. 2007).

Si bien se han realizado estudios botánicos en el departamento de Lima en general y en la provincia de Canta en particular, como los trabajos de Vilcapoma (1975) sobre la familia Solanáceas; Flores (1997) en Fabáceas y Teixeira et al. (2004, 2006) en Cactáceas. No se conocen estudios específicos sobre Poaceae para la zona. En la última década Alegría y Rúgolo de Agrazar (2001) reportan una nueva especie de Muhlenbergia. Los otros trabajos conocidos para Canta (De la Cruz et al., 2005; Espinoza, 1997), no son de tipo taxonómico. Por ello esta investigación tiene por objetivo contribuir al conocimiento de la diversidad de la familia Poaceae en el distrito Arahuay, que sirva de base para un estudio a nivel departamental y nacional.

\section{Área de estudio}

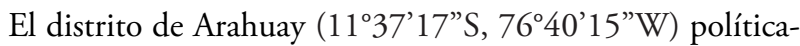
mente pertenece a la provincia de Canta, departamento de Lima. Se encuentra ubicado las vertientes occidentales de los Andes centrales del Perú entre los 1300-4700 msnm. Consideramos como zona baja y media el rango altitudinal comprendido entre los $1300 \mathrm{~m}$ y los $3000 \mathrm{~m}$, la cual tiene una superficie aproximada de $134,29 \mathrm{~km}^{2}$ (Figura 1). El rango altitudinal abarca diversos centros poblados y caseríos entre los más importantes tenemos a Orobel ubicada a $1450 \mathrm{~m}$ de altitud, Licahuasi (1700-2000 m), Arahuay (2500-2900 m), Sinchipampa (2700-3000 m), Tranca $(2700-3000 \mathrm{~m})$.

\section{Materiales y métodos}

Para determinar la riqueza de especies se realizó la recolecta completa en el ámbito del distrito. La recolecta, herborización y

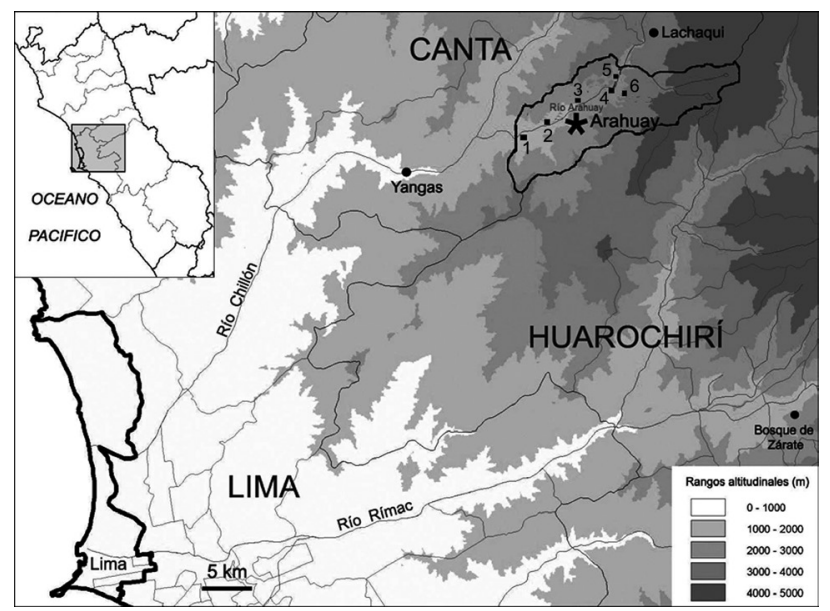

Figura 1. Mapa de ubicación del distrito de Arahuay (Canta, Lima, Perú). Localidades (1) Orobel, (2) Licahuasi, (3) Collo, (4) Arahuay, (5) Tranca, (6) Sinchipampa. 
manejo posterior de especímenes de plantas vasculares siguió las técnicas recomendadas por Cerrate (1969). El mayor esfuerzo de colecta se realizo entre el 05 y el 10 de abril de 2010 .

Las muestras botánicas colectadas se determinaron con el uso de claves botánicas, teniendo como principal referencia el trabajo de Tovar (1993), Las Gramíneas (Poaceae) del Perú, y otras publicaciones especializadas (Renvoize et al. 1998; La Torre et al. 2003, 2004; Valencia et al. 2006) También se consultó las colecciones de del Herbario San Marcos (USM) del Museo de Historia Natural de la Universidad Nacional Mayor de San Marcos, así como consultas a otros especialistas en la familia. La totalidad de la colección botánica ha sido depositada en el herbario San Marcos (USM).

Caracteres Taxonómicos de la familia Poaceae.- Plantas anuales o perennes, comúnmente herbáceas a veces leñosas, cespitosas, rizomatosas o estolonífera; con pocos o numerosos entrenudos y nudos, los entrenudos huecos o sólidos; hojas dísticas sin peciolo, vaina presente; lígula situada entre la vaina y la lámina, membranácea o pubescente, raramente ausente, a veces con dos lóbulos laterales o aurículas; lamina comúnmente lineal, plana o enrollada; inflorescencia en el ápice de las cañas y sus ramificaciones terminales o axilares, abiertas o contraídas, en espiga, racimo o panoja; la espiguilla es la unidad básica de la inflorescencia, muy diversa en estructura, pero típicamente consistiendo en un eje que es la raquilla, que lleva en su base dos brácteas estériles llamadas glumas, de diverso tamaño y consistencia; al interior uno o más antecios o flósculos, consistentes de una bráctea exterior, la lema y otra interior, la palea, esta ultima envuelve a la flor en sí que puede ser hermafrodita o unisexual, con el perianto reducido o excrecencias escamosas turgentes llamadas lodículas; el ovario es unilocular con dos estigmas plumosos; estambres comúnmente tres, fruto usualmente cariópside.

\section{Resultados}

Se registró, para la zona baja y media del distrito de Arahuay, un total de 49 especies de la familia Poaceae agrupadas en 28 géneros, 14 tribus y cuatro subfamilias (Tabla 1, Fig. 1). Las subfamilias con mayor número de especies son Pooideae y Chloridoideae con 21 y 14 especies respectivamente, seguidas de Panicoideae y Arundinoideae con 10 y cuatro especies. Las tribus con mayor número de especies son Paniceae y Poeae con cinco géneros y 10 especies cada una, mientras las tribus Aristideae,

Tabla 1. Subfamilias, Tribus, y especies de la familia Poaceae en el distrito de Arahuay - Canta (Lima- Perú).

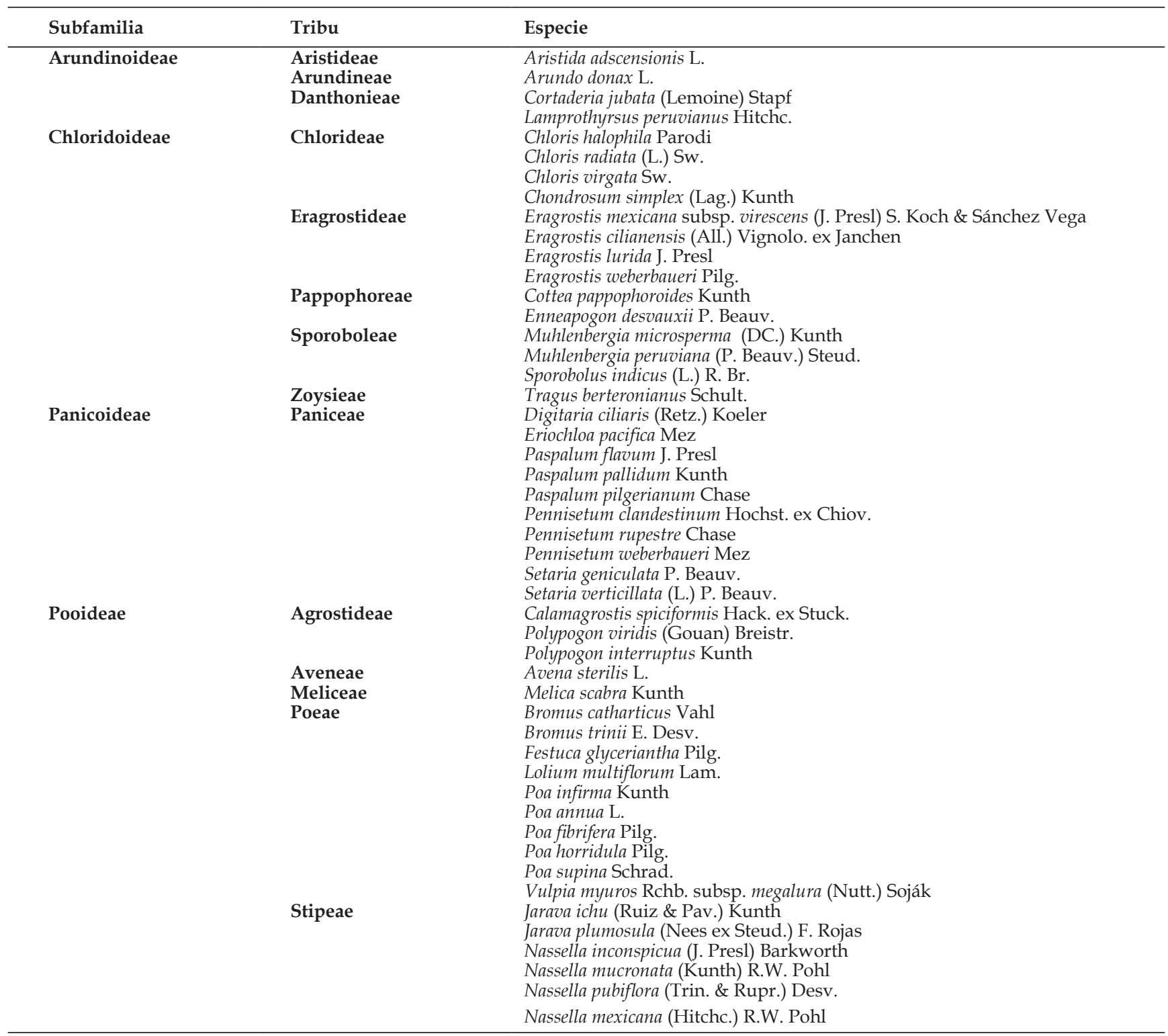


Arundineae, Aveneae, Meliceae y Zoysieae están representadas por solo un género y una especie en cada tribu.

El género Poa es el más diverso con cinco especies, seguido por Eragrostis y Nassella con cuatro especies cada una, mientras que los géneros con menor número de especies fueron Aristida, Arundo, Avena, Chondrosum, Cortaderia, Cottea, Digitaria, Enneapogon, Eriochloa, Festuca, Lolium, Melica, Nassella, Sporobolus, Tragus, Vulpia, todos con sólo una especie.

De las 49 especies que son reportadas, seis constituyen nuevos registros para el departamento de Lima (León et al. 2007: La Torre et al. 2007). Estos son: Calamagrostis spiciformis Hack. ex Stuck., Chloris halophila Parodi, Lamprothyrsus peruvianus Hitchc., Festuca glyceriantha Pilg., Lolium multiflorum Lam. y Poa supina Schrader.

Clave para determinar los géneros de la familia Poaceae del distrito de Arahuay

1a. Raquilla articulada por debajo de las glumas, que caen junto con los granos. 2a. Espiguillas siempre unifloras, solitarias sobre el raquis, caen con el pedicelo. Polypogon

2b. Espiguillas bifloras o multifloras, si uniflora entonces sin las características arriba mencionadas.

3a. Espiguillas multifloras con una a dos flores fértiles y una a dos lemas estériles terminales, las espiguillas triangulares sobre pedicelos flexuosos, blanquecinos o purpúreos.

Melica

3b. Espiquillas 1-2 flores pero sola la flor superior fértil y la inferior masculina o estéril, espiguillas comúnmente dorsiventralmente comprimidas.

4a. Espiguillas rodeadas por una o varias cerdas libres o soldadas en la base formando un involucro.

5a. Cerdas de las espiguillas caducas, delgadas suaves algunas veces plumosas no soldadas en la base. Pennisetum

5b. Cerdas de las espiguillas persistentes en el raquis de modo que las espiguillas caen solas en la madurez.

Setaria

4b. Espiguillas sin cerdas involucrales en su base inflorescencia en espiga o racimo espiciforme unilateral, estos digitados o esparcidos a lo largo del raquis.

6a. Lema fértil endurecida, fuertemente cartilaginosa con los márgenes enrollados sobre la pálea.

7a. Espiguillas con una dilatación o callosidad anular- cupuliforme o discoidea en la base. Eriochloa

7b. Espiguillas sin tal dilatación en la base, con la gluma inferior normal o sin ella. Paspalum

6b. Lema fértil membranácea o ligeramente cartilaginosa con los márgenes no enrollados sobre la palea. Digitara

1b. Raquilla articulada por encima de las glumas, que son persistentes después de la caída de los granos.

8a. Inflorescencia en racimo espiciforme, denso, cilíndrico, gluma superior con pelos gruesos rígidos rectos o uncinados. Tragus

8b. Inflorescencia en panícula abierta o contraída. Gluma superior sin pelos gruesos rígidos erectos o uncinados.

9. Espiguilla uniflora.

10a. Lema con arista simple.

11a. Glumas más largas que la lema, agudas múticas, lema con arista apical.

12a. Lema endurecida rígida o papirácea con antopodio pubescente agudo y punzante.

13a. Lema fusiforme, terete con arista algo vilosa en la parte basal y plumosa hacia la parte media y superior o si no entonces el ápice de la lema con anillo de pelos semirrígidos más largos que la lema y glumas membranáceas. Jarava

13b. Lema fusiforme u obovada, sin la combinación de caracteres anteriores. Nassella

12b. Lema membranácea sin arista o con arista dorsal o subapical, antopodio no pungente.

Calamagrostis

11b. Glumas más cortas que la lema, rara vez mayores, múticas o aristadas, lema con arista apical o subapical

14a. Lemas agudas comúnmente aristadas arista.

14b. Lemas obtusas múticas.

10b. Lema con arista trífida en el ápice.

\section{Muhlenbergia}

Sporobolus

Aristida

9b. Espiguillas bi a multifloras.

15a. Inflorescencias en panículas abiertas o contraídas.

16a. Plantas herbáceas comúnmente menores de 1 metro de altura, espiguillas con lema y raquila glabra o escasamente pubescente. 17a. Lemas mucronadas o aristadas.

18a. Lema no mucronadas.

Avena

18b. Lema mucronada con arista apical o subapical.

19a. Glumas desiguales, lema con arista apical o subapical mucronada; bilobado o bidentada.

20a. Lema aquillada.

21a. Lema múticas raramente aristada, 5-7 nervadas.

21b. Lema mucronada o aristada, 8-11 nervadas.

Festuca

Bromus

20b.Lema no aquillada 5 - multiaristada.

22a. Raquilla fácilmente desarticulable entre las flores y encima de las glumas, redondeadas en el dorso, con cerdas en el borde y ápice de la lema.

Cottea

22b. Raquilla no desarticulable entre las flores todas las flores caen juntas a la madurez, lem truncada y con cerdas ciliadas en el ápice. Enneapogon

19b. Glumas muy desiguales, la inferior a veces muy pequeña, lema en el ápice con arista larga y recta. Vulpia

17b. Lemas múticas o cortamente aristadas.

23a. Inflorescencia en panícula, contraída o abierta.

24a. Paleas persistentes en la raquilla después de caer las glumas y lemas. Eragrostis

24b. Paleas no persistentes en la raquilla después de caer las glumas y lemas.

23b. Inflorescencia en racimos y espigas.

25a. Racimos tipicamente digitados. Espiguillas 2 - 4 flores subsésiles. Solo el antecio basal perfecto de 1 a 2 antecios rudimentarios neutros o masculinos. Lema cortamente aristadas arista.

Chloris

25b. Racimos no digitados. Espiguillas con un antecio basal perfecto 1-4 antecios apicales estériles lema 3 nervada, los nervios extendidos en una arista. Chondrosum

16b. Plantas leñosas o subleñosas mayores de 1 metro de altura, espiguillas con lema y raquila cubierto por largos pelos sedosos.

26a. Lema con arista implantada entre los dos lóbulos o dientes en que se divide el ápice.

Lamprothyrsus

26b. Lema acuminada o subulada no bidentada.

27b. Espiguillas con flores hermafroditas.

27b. Espiguillas con flores unisexuales (dioicas).

15b. Inflorescencia en racimos dístico solitario en el ápice de la caña, espiguilla lateral con una sola gluma.

\section{Géneros y especies de Poaceae en el distrito de Arahuay}

\section{Aristida L.}

Este género agrupa cerca de 250 especies de zonas tropicales y subtropicales. En el Perú se encuentran registradas 12 especies. Para la área estudiada solo una especie.

\section{Aristida adscensionis L.}

En el área estudiada se encuentra entre los 1400-2400 m de altitud correspondientes a la zona baja del distrito, con una mayor abundancia en las comunidades de cactáceas.

Material estudiado: P. Gonzáles 830 (Orobel, 1450 m, 05 abril 2010).

\section{Arundo L.}

Comprende tres especies. Región mediterránea hasta la China. Introducida en América. En el Perú solo se registra una especie ampliamente naturalizada.

\section{Arundo donax L.}

En el área de estudio se encuentra en la ribera de las quebradas, siendo un elemento importante del monte ribereño por debajo de los $2000 \mathrm{~m}$.

\section{Avena L.}

Comprende 25 especies distribuidas principalmente del Mediterráneo y Medio Oriente, existiendo al norte de Europa. Introducida en otras regiones. En el Perú se encuentran presentes cuatro especies, y en la zona de estudio una. 


\section{Avena sterilis $\mathrm{L}$.}

En el área de estudio se encuentra en altitudes intermedios entre los 2400-2700 m correspondientes a la zona media del distrito, en lugares húmedos como el matorral y el monte ribereńo.

Material estudiado: P. Gonzáles 1031 (Arahuay, 2500-2650 m, 08 abril 2010).

\section{Chondrosum Desv.}

Comprende cerca de 40 especies y es originario de America donde se distribuye desde Canadá hasta Sudamerica con la mayor cantidad de especies en Estados Unidos. En el Perú solo se registra una especie.

\section{Chondrosum simplex (Lag.) Kunth}

En el lugar de estudio fue enontrado en lugares moderadamente secos por debajo de los $2400 \mathrm{~m}$.

Material estudiado: P. Gonzáles 1040 (Arahuay, 2500-2650 m, 08 abril 2010).

\section{Bromus L.}

Comprende alrededor de 150 especies distribuidas en regiones templadas de ambos hemisferios, pero principalmente en el norte. En el Perú se reconocen 8 especies.

\section{Claves para las especies del género Bromus}

1a. Lemas conspicuamente dentadas, los dientes de 2 a tres $\mathrm{mm}$ de largo, con arista retorcida. B. trinii

1b. Lemas múticas o ligeramente dentadas, en este caso los dientes menores a

$1 \mathrm{~mm}$ de largo con arista recta. B. catharticus

5. Bromus catharticus Vahl

Observado en lugares secos a moderadamente húmedos, cercano al monte ribereńo por encima de los $2400 \mathrm{~m}$.

Material estudiado: P. Gonzáles 021 (Quebrada del Rio Chico, 2450 m, 20 marzo 2008); 637 (Quebrada del Rio Chico, 2450 m, 27 octubre 2009); 1161 (Tranca, 2800-3200 m, 10 abril 2010).

\section{Bromus trinii E. Desv.}

Habita lugares sombreados, se encuentra mayormente en lugares moderadamente húmedos, estando presente en la formación de matorral y monte ribereño a altitudes de $2600 \mathrm{~m}$.

Material estudiado: P. Gonzáles 1036 (Arahuay, 2500-2650 m, 08 abril 2010); 1157 (Tranca, 2800-3200 m, 10 abril 2010).

\section{Calamagrostis Adans.}

Este género agrupa cerca de 270 especies, en regiones templadas y también en las montañas de los trópicos. En el Perú está representada por 43 especies.

\section{Calamagrostis spiciformis Hack. ex Stuck.}

En la zona de estudio se encuentra en lugares secos, encontrado en la formación de matorral por encima de los $3000 \mathrm{~m}$.

Material estudiado: P. Gonzáles 1098 (Tranca, 2800-3200 m, 10 abril 2010)

\section{Chloris Sw.}

Este género comprende más o menos 40 especies ampliamente distribuidas en los trópicos y partes cálidas de las zonas templadas. Para la flora peruana se reportan 6 especies y tres en la zona de estudio.

\section{Claves para las especies del género Chloris}

1a. Plantas perenne, estolonífera, laminas foliares obtusa. C. halophila

1b. Plantas anuales, no estolonífera.

2a. Lema fértil algo ancha gibosa en el dorso. $\quad$ C. virgata

2a. Lema terete o fusiforme. $\quad$ C. radiata

8. Chloris halophila Parodi

Se ha encontrado en lugares secos a moderadamente secos entre los 1300 y $1600 \mathrm{~m}$. Su presencia es escasa.

Material estudiado: P. Gonzáles 946 (Licahuasi, 1900 m, 06 abril 2010).

\section{Chloris radiata (L.) Sw.}

Su hábitat son los campos abiertos, áreas alteradas y campos de cultivo, como maleza. En el área de estudio se encuentra en lugares secos a moderadamente secos entre los 1300 y $1600 \mathrm{~m}$. Su presencia es moderada.

Material estudiado: P. Gonzáles 875 (Orobel, 1450 m, 05 abril 2010).

\section{Chloris virgata Sw.}

Se encuentra en lugares secos a moderadamente secos entre los 1300 y 1600 m. Es muy abundante.

Material estudiado: P. Gonzáles 876 (Orobel, 1450 m, 05 abril 2010).

\section{Cortaderia Stapf}

Este género agrupa cerca de 24 especies, la mayoria de Sudamérica, 4 especies en Nueva Zelanda y una en Nueva Guinea. En el Perú se reportan 8 especies y en la zona de estudio una.

\section{Cortaderia jubata (Lemoine) Stapf}

Se ha reportado en lugares húmedos por encima de los 2800 $\mathrm{m}$ formando parte del monte ribereño.

Material estudiado: P. Gonzáles 086 (Arahuay, 2750 m, 23 marzo 2008); 1159 (Tranca, 2800-3200 m, 10 abril 2010).

\section{Cottea Kunth}

Comprende una especie distribuida desde el sur de los Estados Unidos de Norteamérica hasta Argentina en las planicies y cerros secos.

\section{Cottea pappophoroides Kunth}

Se encuentra en lugares moderadamente secos formando parte del estrato inferior del piso de cactáceas, a altitudes de $1400 \mathrm{~m}$.

Material estudiado: P. Gonzáles 929 (Licahuasi 1900 m, 06 abril 2010).

\section{Digitaria Haller}

Comprende doscientas especies tropicales, subtropicales y zonas cálidas de las regiones templadas. En el Perú se encuentran 13 especies y una en la zona de estudio.

\section{Digitaria ciliaris (Retz.) Koeler}

Se encuentra en lugares húmedos por debajo de los 1800 $\mathrm{m}$ formando parte del monte ribereńo. Su presencia es escasa.

Material estudiado: P. Gonzáles 874 (Orobel, 1450 m, 05 abril 2010).

\section{Enneapogon P. Beauv.}

Este género comprende 28 especies de los trópicos y subtrópicos del mundo, principalmente en Australia y África. En 
la flora peruana y la zona de estudio una.

\section{Enneapogon desvauxii P. Beauv.}

En el distrito se encuentra en lugares moderadamente secos formando parte del estrato inferior del piso de cactáceas, a altitudes de $1400 \mathrm{~m}$. Su presencia escasa.

Material estudiado: P. Gonzáles 840 (Orobel, 1450 m, 05 abril 2010).

\section{Eragrostis Wolf}

Este género agrupa más o menos 350 especies de los trópicos y subtrópicos del mundo. En el Perú se conocen 29 especies y cuatro en la zona de estudio.

\section{Claves para las especies del género Eragrostis}

1a. Plantas anuales, panoja abierta.

2a. Espiguillas de 2- $4 \mathrm{~mm}$ de ancho. E. cilianensis

2b. Espiguillas de menores de $1.8 \mathrm{~mm}$ de ancho. E. mexicana subsp. virescens

1b. Plantas perennes, panoja algo angosta o contraídas

3a. Ramas de la panoja ascendentes o algo divergentes.

3b. Ramas de la panojas adpresas.

E. weberbaueri

15. Eragrostis cilianensis (All.) Vignolo. ex Janch.

Está presente en lugares moderadamente secos formando parte del estrato inferior del piso de cactáceas, entre las altitudes de 1300-1600 m. Es muy abundante.

Material estudiado: P. Gonzáles 842 (Orobel, 1450 m, 05 abril 2010).

\section{Eragrostis lurida J. Presl}

Se encuentra en lugares húmedos formando parte del matorral, a altitudes superiores a $2600 \mathrm{~m}$. Su presencia es moderada.

Material estudiado: P. Gonzáles 1155 (Tranca, 2800-3200 m, 10 abril 2010.

17. Eragrostis mexicana subsp. virescens (J. Presl) S.D. Koch \& Sánchez Vega

Está presente en lugares secos moderadamente húmedos. Está ampliamente distribuída.

Material estudiado: P. Gonzáles 845 (Orobel, 1450 m, 05 abril 2010). 1042 (Arahuay, 2500-2650 m, 08 abril 2010).

\section{Eragrostis weberbaueri Pilg.}

Se encuentra en laderas pedregosas secas, está ampliamente distribuída.

Material estudiado: P. Gonzáles 969 (Camino a Sinchipampa, 2600-3100 msnm. 07 abril 2010).

\section{Eriocloa Kunth}

Comprende 30 especies distribuidas a través de los trópicos. Para la flora peruana se registran nueve especies y una para la zona de estudio.

\section{Eriochloa pacifica $\mathrm{Mez}$}

En el área de estudio se encuentra en lugares moderadamente secos, a altitudes de $1400 \mathrm{~m}$, con escasa presencia.

Material estudiado: P. Gonzáles 931 (Licahuasi 1900 m, 06 abril 2010).

\section{Festuca L.}

Género que comprende más o menos 450 especies, de regiones templadas extendiéndose a las montańas de los trópicos. En el Perú se han registrado 42 especies y para la zona de estudio una.

\section{Festuca glyceriantha Pilg.}

Se ha encontrado en lugares moderadamente secos, a altitudes mayores a $3000 \mathrm{~m}$, con escasa presencia.

Material estudiado: P. Gonzáles 1160 (Tranca, 2800-3200 m, 10 abril 2010).

\section{Jarava Ruiz \& Pav.}

Género con aproximadamente 300 especies de zonas templadas y templado-cálidas del mundo, anteriormente tratadas como Stipa. En el Perú se reportan 13 especies y dos en la zona de estudio.

\section{Claves para las especies del género Jarava}

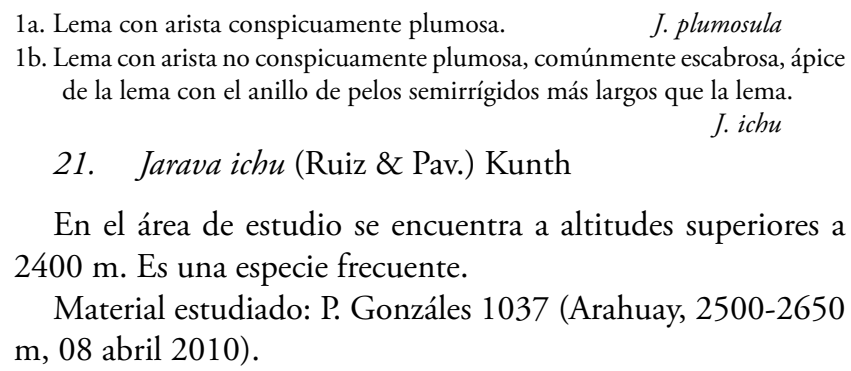

\section{Jarava plumosula (Nees ex Steud.) F. Rojas}

Muy difundido en los departamentos andinos. En el área de estudio se encuentra a altitudes medias de $2400 \mathrm{~m}$. Su presencia es frecuente.

Material estudiado: P. Gonzáles 1039 (Arahuay, 2500-2650 m, 08 abril 2010).

\section{Lamprothyrsus Pilg.}

Comprende únicamente tres especie presentes en Perú, Bolivia y Argentina. En la zona de estudio una.

\section{Lamprothyrsus peruvianus Hitchc.}

Se encuentra a altitudes medias de $2800 \mathrm{~m}$. Es una especie escasa.

Material estudiado: P. Gonzáles 666 (Quebrada del rio Chico, 2450 m, 27 octubre 2009).

\section{Lolium L.}

Consta de ocho especies propias de Eurasia, introducidas en otras partes del mundo. En el Perú se reportan 3 especies y en la zona de estudio una.

\section{Lolium multiflorum Lam.}

En el área de estudio se encuentra en el monte ribereño, a altitudes superiores a $2800 \mathrm{~m}$. Su presencia es moderada.

Material estudiado: P. Gonzáles 1156 (Tranca, 2800-3200 m, 10 abril 2010).

\section{Melica L.}

Comprende más o menos 80 especies de regiones templadas. En el Perú y la zona de estudio una.

\section{Melica scabra Kunth}

Está presente formando parte del matorral sobre los $2500 \mathrm{~m}$. Es una especie frecuente.

Material estudiado: P. Gonzáles 1033a (Arahuay, 2500-2650 m, 08 abril 2010). 


\section{Mublenbergia Schreb.}

Abarca más o menos 160 especies la mayoría son especies americanas. En el Perú se reportan 13 especies y la zona de estudio dos.

\section{Claves para las especies del género Mublenbergia}

1a. Planta anual, gluma superior 3 dentada, la arista flexuosa. M. peruviana

1b. Planta anual, gluma superior entera, la arista recta. M. microsperma

26. Muhlenbergia microsperma (DC.) Kunth

En el área de estudio se encuentra formando parte del matorral y monte ribereño entre los 1600 y $2800 \mathrm{~m}$. Es una especie frecuente.

Material estudiado: P. Gonzáles 930 (Licahuasi 1900 m, 06 abril 2010).

\section{Mublenbergia peruviana (P. Beauv.) Steud.}

Es frecuente. En la zona de estudio está ampliamente distribuido en la zona media entre 2300 y $3600 \mathrm{~m}$.

Material estudiado: P. Gonzáles 1040 (Arahuay, 2500-2650 m, 08 abril 2010)

\section{Nassella (Trin) Desv.}

Este género comprende 109 especies, varias especies han sido segregadas del género Stipa, todas son nativas del nuevo mundo. En el Perú se han registrado 16 especies y la zona de estudio cuatro.

\section{Claves para las especies del género Nassella}

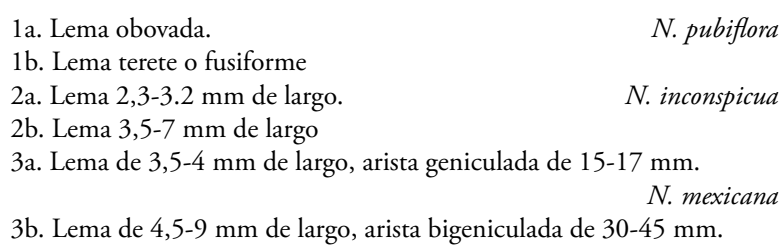

28. Nassella inconspicua (J. Presl) Barkworth

Observado a altitudes superiores a los $2400 \mathrm{~m}$.

Material estudiado: P. Gonzáles 1038 (Arahuay, 2500-2650 m, 08 abril 2010).

\section{Nassella mexicana (Hitchc.) R.W. Pohl}

En el área de estudio se encuentra en altitudes superiores a $2800 \mathrm{~m}$.

Material estudiado: P. Gonzáles 1162 (Tranca, 2800-3200 m, 10 abril 2010).

\section{Nassella mucronata (Kunth) R.W. Pohl}

Habita lugares con altitudes superiores a $2600 \mathrm{~m}$, generalmente en laderas empinadas.

Material estudiado: P. Gonzáles 1158 (Tranca, 2800-3200 m, 10 abril 2010).

\section{Nassella pubiflora (Trin. \& Rupr.) Desv.}

En el área de estudio se encuentra en el monte ribereńo, a altitudes superiores a $2800 \mathrm{~m}$. Es una especie frecuente.

Material estudiado: P. Gonzáles 1163 (Tranca, 2800-3200 m, 10 abril 2010).

\section{Paspalum L.}

Género con aproximadamente 330 especies a través de los trópicos, pero la mayoría de América. En el Perú se reportan 49 especies y la zona de estudio tres.

\section{Claves para las especies del género Paspalum}

1a. Anual, racimos algo arqueados, espiguillas $2-2.3 \mathrm{~mm}$. P. flavum 1b. Perenne, racimos ascendentes, espiguillas 2,3-3 mm.

2a. Espiguillas aovado-oblongas, de 2.3-2.5 mm de largo, raquis $1.5-2 \mathrm{~mm}$. P. pilgerianum

2b Espiguillas oblongo-elípticas, de $2.8-3 \mathrm{~mm}$ de largo, raquis $2.3-2.8 \mathrm{~mm}$.

\section{Paspalum flavum J. Presl} P. pallidum

En el área de estudio se encuentra a altitudes medias de 2400 $m$. Es una especie frecuente.

Material estudiado: P. Gonzáles 966 (Camino a Sinchipampa, 2600-3100 msnm. 07 abril 2010). 667 (Quebrada del rio Chico, $2450 \mathrm{~m}, 27$ octubre 2009).

\section{Paspalum pallidum Kunth}

Habita los campos abiertos del área de estudio se encuentra entre 2400 y $3000 \mathrm{~m}$. Es una especie frecuente.

Material estudiado: P. Gonzáles 1167 (Tranca, 2800-3200 m, 10 abril 2010).

\section{Paspalum pilgerianum Chase}

Habita los campos abiertos. En el área de estudio se encuentra entre 2800 y $3000 \mathrm{~m}$. Es una especie frecuente.

Material estudiado: P. Gonzáles 1030 (Arahuay, 2500-2650 m, 08 abril 2010); 1096 (Tranca, 2800-3200 m, 10 abril 2010).

\section{Pennisetum Pers.}

Comprende cerca de 70 especies distribuida en los trópicos y subtrópicos. En el Perú se han reportado 18 especies y la zona de estudio tres

\section{Claves para las especies del género Pennisetum}

1a. Plantas estolonífera, rastrera, espigas poco visibles inclusas en las vainas. P. clandestinum

1b. Plantas perennes, cespitosa erguida, espigas excertas.

2a. Espigas de 6.2- $8 \mathrm{~mm}$ de largo, inflorescencias pocas rígida. P. rupestre

2b. Espigas menores a 5.5-6.5 mm de largo, inflorescencias numerosas, flex-

uosas. P. weberbaueri

35. Pennisetum clandestinum Hochst. ex Chiov.

En el área de estudio se encuentra a altitudes medias de 2400 m. En las cercanías a los poblados es frecuente.

Material estudiado: P. Gonzáles 653 (Quebrada del rio Chico, 2450 m, 27 octubre 2009).

\section{Pennisetum rupestre Chase}

Se le encuentra en campos abiertos algo secos a altitudes medias de $2400 \mathrm{~m}$. Es una especie frecuente.

Material estudiado: P. Gonzáles 1034 (Arahuay, 2500-2650 m, 08 abril 2010).

\section{Pennisetum weberbaueri Mez}

Habita en campos abiertos, entre arbustos semicaducifolio, de suelos algo secos. En el área de estudio se encuentra a altitudes medias de $2400 \mathrm{~m}$. Es una especie frecuente.

Material estudiado: P. Gonzáles 1035 (Arahuay, 2500-2650 m, 08 abril 2010).

Poa L.

Género con 500 especies, propias de las regiones templadas 
y frías de ambos hemisferios. En el Perú se reportan 41 especies y la zona de estudio cinco

\section{Claves para las especies del género Poa}

1a. Plantas anuales

2a. Lema glabras o algo pubescentes en los nervios, cańa algo engrosada. P. supina

2b. Lema pubescentes en la mitad inferior, caña delgada

3a. Lemas angostas, oblongo-lanceoladas; raquilas alargadas. P. infirma

3b. Lemas ensanchadas aovado-lanceoladas; raquilas alargadas. P. annua

1b. Plantas perennes

4a. Lemas escabrosa. P. fibrifera

4b. Lemas viloso-pubescentes en la mitad inferior. $\quad P$. horridula

38. Poa annua L.

En el área de estudio se la ha encontrado desde los 1500 hasta los $3000 \mathrm{~m}$. Es una especie frecuente.

Material estudiado: P. Gonzáles 003 (Quebrada del rio Chico, 2450 m, 20 marzo 2008); 1166 (Tranca, 2800-3200 m, 10 abril 2010).

\section{Poa fibrifera Pilg.}

En el área de estudio solo se la ha encontrado a los $2800 \mathrm{~m}$. Es una especie frecuente.

Material estudiado: P. Gonzáles 1032 (Arahuay, 2500-2650 m, 08 abril 2010).

\section{Poa horridula Pilg.}

En el área de estudio se la ha encontrado en el monte ribereño y zonas aledańas a esta formación, por sobre los $2800 \mathrm{~m}$.

Material estudiado: P. Gonzáles 1165 (Tranca, 2800-3200 m, 10 abril 2010).

\section{Poa infirma Kunth}

Observada en el monte ribereño, por encima de los $2800 \mathrm{~m}$.

Material estudiado: P. Gonzáles 1164 (Tranca, 2800-3200 m, 10 abril 2010).

\section{Poa supina Schrad.}

En el área de estudio se encuentra a altitudes medias de 2400 $m$. Es una especie frecuente.

Material estudiado: P. Gonzáles 1033b (Arahuay, 2500-2650 m, 08 abril 2010).

\section{Polypogon Desf.}

Dieciocho especies conforman este género, de regiones templadas cálidas del mundo y también en las montañas tropicales. En el Perú se reportan 5 especies y la zona de estudio dos.

\section{Claves para las especies del género Polypogon}

1a. Glumas múticas.

1b. Glumas largamente aristada.

43. Polypogon interruptus Kunth

Habita suelos húmedos. En el área de estudio se encuentra en la ribera de quebradas, desde los 1400 hasta los $3200 \mathrm{~m}$.

Material estudiado: P. Gonzáles 1054 (Arahuay, 2500-2650 m, 08 abril 2010).

\section{Polypogon viridis (Gouan) Breistr.}

En el área de estudio se encuentra formando parte del monte ribereño a altitudes de $1600 \mathrm{~m}$. Es una especie frecuente.

Material estudiado: P. Gonzáles 877 (Orobel, 1450 m, 05 abril 2010).

\section{Setaria P. Beauv.}

Cuenta con cien especies de los trópicos y subtrópicos en ambos hemisferios. En el Perú se registran 15 especies y la zona de estudio dos.

\section{Claves para las especies del género Setaria}

1a. Espiguillas rodeadas en la base por cinco o más setas. S. geniculata 1b. Espiguillas rodeadas en la base por una sola seta. S. verticillata

45. Setaria geniculata P. Beauv.

En el área de estudio se encuentra formando parte del monte ribereño a altitudes de $1400 \mathrm{~m}$. Es una especie frecuente.

Material estudiado: P. Gonzáles 872 (Orobel, 1450 m, 05 abril 2010).

\section{Setaria verticillata (L.) P. Beauv.}

Se encuentra formando parte del monte ribereño a altitudes de $1400 \mathrm{~m}$. Es una especie abundante.

Material estudiado: P. Gonzáles 873 (Orobel, 1450 m, 05 abril 2010).

\section{Sporobolus R. Br.}

Género que abarca cerca de 160 especies de los trópicos y subtrópicos de ambos hemisferios. En el Perú se reportan 10 especies y la zona de estudio una.

\section{Sporobolus indicus (L.) R. Br.}

Muy común en campos abiertos. Se le ha encontrado en el monte ribereño, por encima de los $2800 \mathrm{~m}$.

Material estudiado: P. Gonzáles 1154 (Tranca, 2800-3200 m, 10 abril 2010).

\section{Tragus Haller}

Constituida por siete especies ampliamente distribuida en los trópicos. En el Perú se reportan 2 especies y para la zona de estudio una.

48. Tragus berteronianus Schult.

Ha sido encontrado en el piso de las cactáceas a los $1400 \mathrm{~m}$.

Material estudiado: P. Gonzáles 844 (Orobel, 1450 m, 05 abril 2010).

\section{Vulpia C.C. Gmel.}

Abarca 22 especies, propias de las regiones templadas, y subtropicales de los hemisferios Norte y Sur. En el Perú se reportan 4 especies y la zona de estudio una.

49. Vulpia myuros Rchb. subsp. megalura (Nutt.) Soják

Se la encuentra en suelos algo sombreados. En el área de estudio se le ha encontrado en las partes altas sobre los $2800 \mathrm{~m}$.

Material estudiado: P. Gonzáles 1054 (Arahuay, 2500-2650 m, 08 abril 2010).

\section{Discusión y conclusión}

Se elevan a 49 especies de Poaceae de las 15 especies reportadas anteriormente por Espinoza (1997) en la zona de estudio, debido a que este último solo estudió las especies etnobotánicas.

Nuestros resultados son comparables con el trabajo de Valencia et al. (2006), quienes reportan para el Bosque de Zárate (Huarochirí) 44 especies en 22 géneros, en un rango altitudinal de 1500 a $3400 \mathrm{~m}$, cabe indicar que estas dos zonas son muy 
similares (Fig. 1), llegando a tener las mismas formaciones vegetales y condiciones climáticas.

La mayoría (seis especies) que se consideran nuevos registros para el departamento de Lima (excepto Poa supina) han sido colectados en áreas poco o casi nada perturbadas, lo cual nos indica que su distribución es natural. Estos nuevos registros básicamente se deben a la falta de estudios en el área, ya que se tiene reporte de estas especies en departamentos cercanas a lima. Estas novedades pueden deberse a que en este estudio se realizó una colecta más intensiva y en localidades anteriormente no visitadas.

De las 15 especies endémicas presentes en el departamento de Lima (La Torre et al. 2007), sólo Pennisetum rupestre se encuentra en el área de estudio. Esta especie también está reportada en otros departamentos del Perú tales como Cajamarca y Junín.

En comparación Valencia et al. (2006), en este estudio se ha encontrado cinco especies más, además el rango altitudinal de nuestra área de estudio es ligeramente menor, por lo tanto Podemos concluir que el distrito Arahuay (zonas baja y media) tiene una alta diversidad de Poaceae con 49 especies comprendidas en 28 géneros, 14 tribus y 4 subfamilias y que representan el 33\% de las especies registradas para el departamento de Lima.

\section{Agradecimientos}

Queremos agradecer a Blanca León por la revisión del manuscrito y sus importantes sugerencias; así como a José Roque por la elaboración del mapa de ubicación del área de estudio.

\section{Literatura citada}

Alegria J. \& Z. Rúgolo de Agrazar. 2001. Muhlenbergia monandra (Poaceae: Eragrostideae), nueva especie anual endémica del Perú. Darwiniana 39(1-2): 19-28.

Brack A. 1986. Ecología de un país complejo. En Gran Geografía del Perú: Naturaleza y Hombre. Volumen II., Manfer-Mejía Baca, España:175-319.

Brako L. \& J. Zarucchi. 1993. Catalogue of the Flowering Plants and Gymnosperms of Peru. Monogr. Syst. Bot. Missouri Bot. Gard. 45: 1-1286.

Cerrate E. 1969. Manera de preparar Plantas para el Herbario Mus. His. Nat. Serie de Divulgación $\mathrm{N}^{\circ} 1$.

Watson L. \& M. J. Dallwitz. 1992. The Grasses Genera of The World. C. A. B. International, Willingford, England.

De la Cruz H., P. Zevallos \& G. Vilcapoma. 2005. "Status" de conservación de las especies vegetales silvestres de uso tradicional en la provincia de Canta, Lima-Perú. Ecología Aplicada, Vol. 4 No 1 y 2, pp. 9-16.
Espinoza F. 1997. Estudio etnobotánico del distrito de Arahuay (prov. Canta, dpto. Lima). Tesis para optar el grado académico de magister en botánica tropical mención: etnobotánica. Lima - Perú. 189 pp.

Flores M. 1997. La familia leguminosae en el valle del Chillónparte media y alta, Departamento de Lima. Tesis Magíster. Universidad Nacional Mayor de San Marcos. Lima - Perú. $114 \mathrm{pp}$.

La Torre M.I., A. Cano \& O. Tovar. 2003. Las Poáceas del Parque Nacional Yanachaga-Chemillén, Oxapampa, Pasco, Perú. Parte I: Bambusoideae. Rev. perú biol. 10(2): 145 - 154.

La Torre M.I., A. Cano \& O. Tovar. 2004. Las Poáceas del Parque Nacional Yanachaga-Chemillén, Oxapampa, Pasco, Perú. Parte II: Pooideae, Centothecoideae, Arundinoideae, Chloridoideae y Panicoide. Rev. perú biol. 11(1): 51 - 70 .

La Torre M.I., J. Alegría, N. Refulio \& I. Sánchez. 2007. Poaceae endémicas del Perú. En: B. León et al. (ed.). El libro rojo de las especies endémicas del Perú. Rev. per. Biol. Número especial 13(2): 879s - 891s.

León B., J. Roque, C. Ulloa Ulloa, et al. 2007. El libro rojo de las especies endémicas del Perú. Rev. per. biol. Número especial 13(2). Pp. 971.

Renvoize S.A., A. Anton \& S. Beck. 1998. Gramineas de Bolivia. The Royal Botanic Gardens, Kew. Pp. 643.

Teixeira V. 2006. Análisis de la diversidad de la composición de Cactáceas y su relación con los factores edáficos en el valle del río Chillón, Lima: cerro Umarcata y quebrada Orobel. Tesis para optar el Grado Académico de Magíster Scientiae. UNALM. Lima - Perú.

Teixeira V., V. Castro, A. Ceroni \& R. Eyzaguirre. 2004. Diversidad y densidad de la comunidad de cactáceas en el valle del río Chillón: cerro Umarcata y quebrada Orobel y su relación con los factores edáficos. Ecología Aplicada. 3 (1 y 2$): 1-8$.

Tovar O. 1993. Las Gramíneas (Poaceae) del Perú. Ruizia, Tomo 13, Madrid. $481 \mathrm{pp}$.

Valencia N., M.I. La Torre, A. Cano \& O. Tovar. 2006. Las Poáceas del Bosque de Zárate. Zonas bajas y media de la ladera de la margen derecha del río Seco. Boletín de Lima. 144: 95-145

Van Der Werff H. \& T. Conciglio. 2004. Distribution and Conservation significance of endemic species of flowering plants in Perú. Biodiversity and conservation. 13: 1699-1713.

Vilcapoma G. 1975. Las Solanaceae del valle de Chillón provincia de Canta. Tesis para optar el grado de Bachiller. Lima Perú. 178 pp.

Vilcapoma G. 2007. Frutos silvestres (Solanáceas) de la cuenca del río Chillón, provincia de Canta, Lima - Perú, ecología aplicada, 6(1,2): 23-32

Weberbauer A. 1945. El Mundo Vegetal de los Andes Peruanos. Ministerio de Agricultura, Lima. 776 pp. 